\title{
Moderate quality evidence that surgical anchorage more effective than conventional anchorage during orthodontic treatment
}

\author{
Abstracted from \\ Jambi S, Walsh T, Sandler J, Benson PE, Skeggs RM, O'Brien KD. \\ Reinforcement of anchorage during orthodontic brace treatment with implants or other surgical methods. \\ Cochrane Database Syst Rev 2014; 8: Art. No. CD005098. DOI: 10.1002/14651858.CD005098.pub3. \\ Address for correspondence: Luisa Fernandez Mauleffinch, Review Group Co-ordinator, \\ Cochrane Oral Health Group, School of Dentistry, The University of Manchester, \\ Coupland III Building, Oxford Road, Manchester, M13 9PL, UK. E-mail: luisa.fernandez@manchester.ac.uk
}

\section{Question: Are surgical anchorage techniques more effective than conventional anchorage in preventing unwanted tooth movement during orthodontic treatment?}

Data sources Cochrane Oral Health Group's Trials Register, the Cochrane Central Register of Controlled Trials (CENTRAL), Medline, Embase, key international orthodontic and dental journals and the World Health Organization (WHO) International Clinical Trials Registry Platform.

Study selection Randomised controlled trials comparing surgical anchorage with conventional anchorage in orthodontic patients. Trials comparing two types of surgical anchorage were also included. Data extraction and synthesis Data extraction was performed independently and in duplicate by three review authors and the Cochrane risk of bias tool was used to assess bias. Random-effects meta-analysis was used for more than three studies when pooling of the data was clinically and statistically appropriate. Fixed-effect analysis was undertaken with two or three studies.

Results Fifteen studies, involving 543 analysed participants, were included. Five ongoing studies were identified. Eight studies were assessed to be at high overall risk of bias, six at unclear risk and one study at low risk of bias. Ten studies (407 randomised and 390 analysed patients) compared surgical anchorage with conventional anchorage for the primary outcome. A random-effects meta-analysis of seven studies for the primary outcome found strong evidence of an effect of surgical anchorage. Compared with conventional anchorage, surgical anchorage was more effective in the reinforcement of anchorage by $1.68 \mathrm{~mm}(95 \% \mathrm{Cl}-2.27 \mathrm{~mm}$ to $-1.09 \mathrm{~mm}$ ) (moderate quality evidence). This result should be interpreted with some caution, however, as there was a substantial degree of heterogeneity for this comparison. There was no evidence of a difference in overall duration of treatment between surgical and conventional anchorage (low quality of evidence).

Information on patient-reported outcomes such as pain and acceptability was limited and inconclusive. When direct comparisons

This paper is based on a Cochrane Review published in the Cochrane Library 2014, issue 8 (see www.thecochranelibrary.com for information). Cochrane Reviews are regularly updated as new evidence emerges and in response to feedback, and the Cochrane Library should be consulted for the most recent version of the review. were made between two types of surgical anchorage, there was a lack of evidence to suggest that any one technique was better than another. Conclusions There is moderate quality evidence that reinforcement of anchorage is more effective with surgical anchorage than conventional anchorage, and that results from mini-screw implants are particularly promising. While surgical anchorage is not associated with the inherent risks and compliance issues related to extra-oral headgear, none of the included studies reported on harms of surgical or conventional anchorage.

\section{Commentary}

Numerous types of anchorage are used to control the reciprocal forces of orthodontic tooth movement. ${ }^{1}$ In this Cochrane systematic review the effectiveness of surgical anchorage (SA) was compared with conventional anchorage (CA) systems. The former methods were found to be more effective, ie less mesio-distal movement of the maxillary first molar. This outcome has to be considered in the perspective of various forms of heterogeneity in the selected studies, which could both inflate or deflate this value.

First, most patients in the eligible studies were treated in orthodontic departments of universities. Effectiveness in such settings could differ from those recorded in private orthodontic clinics. Second, broad-spectrum eligibility criteria were used for both types of anchorage. For the SA systems, mid-palatal implants, onplants, and mini-screw implants were included, which vary in surgical techniques, sites of insertion, screw characteristics, loading protocols and biomechanics. For the CA systems, headgears, palatal arches and combinations of conventional anchorage systems were eligible. Third, the timing for measuring treatment endpoints varied widely between eligible studies.

These sources of heterogeneity could influence the effectiveness of the different anchorage systems and consequently the external validity of these outcomes. Other factors that could affect the applicability of a specific anchorage method include: 1) the quality rating of the outcome 'effectiveness'. A 'moderate' quality score according to the 'Grading of Recommendations Assessment Development and Evaluation' (GRADE) approach was applied to this outcome, which indicates that 'further research is likely to have an important impact on our confidence in the estimate of effect and may change the estimate'; ${ }^{2,3}$ 2) the magnitude of the outcome. The mean difference of effectiveness, ie, $1.68 \mathrm{~mm}$ (95\% CI $-2.27 \mathrm{~mm}$ to $-1.09 \mathrm{~mm}$ ), was scored in favour of SA. The magnitude of this measure should be considered when selecting a 
specific method of anchorage; 3) the importance of the outcome 'effectiveness' to pertinent stakeholders, eg, clinicians, patients, researchers, guideline developers, policy-makers and implant companies; 4) the potential importance of other outcomes, eg qualitative research data. The assessment of patient-important outcomes such as 'health experiences' was suboptimal in the included studies for both anchorage systems; 5) the time points for measuring outcomes. The short-term outcomes in the selected studies could vary from those recorded in the long run and have a different clinical importance; 6) costs; and 7) adverse effects of interventions. The applicability of SA strongly depends on the costs of the interventional procedures and developing clinical skills and experience. In addition, the balance between desirable outcomes and adverse effects should be carefully weighed. ${ }^{4}$ None of the eligible studies addressed these issues.

This systematic review was conducted by experienced reviewers and topic experts according to the highest standards in evidencebased health care. The 'assessment of multiple systematic reviews' (AMSTAR) instrument was applied to critically appraise this research study. ${ }^{5}$ All 11 questions of this assessment tool were scored with a 'yes' indicating the good methodological quality of this review.

The authors presented a detailed list of implications for clinical practice and research topics for future studies. Measuring both qualitative and quantitative outcomes of surgical and conventional anchorage systems at both short- and long-term endpoints could be the key research direction of choice.

\section{Practice points}

- Surgical anchorage was more effective, $1.68 \mathrm{~mm}(95 \% \mathrm{Cl}-2.27$ $\mathrm{mm}$ to $-1.09 \mathrm{~mm}$ ), than conventional anchorage systems, based on 'moderate' quality evidence. Outcomes with mini-screw anchorage were particularly promising.

- The application of surgical anchorage to clinical practice should be considered in the perspective of the quality and the magnitude of the outcome 'effectiveness', other 'patient-important outcomes', clinical experience, the high heterogeneity and underreporting in the included studies, the costs of interventions and the balance between desirable and non-desirable outcomes.

\section{Reint Meursinge Reynders and Jan de Lange \\ Department of Oral and Maxillofacial Surgery, Academic Medical Center, University of Amsterdam, The Netherlands}

1. Skeggs RM, Benson PE, Dyer F. Reinforcement of anchorage during orthodontic brace treatment with implants or other surgical methods. Cochrane Database Syst Rev 2007; 18;(3): CD005098.

2. Balshem $H$, Helfand $M$, Schünemann $H$, et al. GRADE guidelines: 3 . Rating the quality of evidence. / Clin Epidemiol 2011; 64: 401-406.

3. Criteria for applying or using GRADE. GRADE working group. [online] Available from: http://www.gradeworkinggroup.org/publications/Minimum_criteria_for_using GRADE_web.pdf [Accessed 30 October 2013].

4. Meursinge Reynders RA. Low quality evidence on the stability of orthodontic mini-implants. Evid Based Dent 2013; 14: 78-80.

5. Shea B], Grimshaw JM, Wells GA, et al. Development of AMSTAR: a measurement tool to assess the methodological quality of systematic reviews. BMC Med Res Methodol 2007; 7: 10 .

Evidence-Based Dentistry (2014) 15, 108-109. doi:10.1038/sj.ebd.6401060 